\title{
AVALIAÇÃO DA QUALIDADE DE VIDA DE IDOSOS PRATICANTES DE ATIVIDADE FÍSICA DE UMA UNIDADE BÁSICA DE SAÚDE DE MINAS GERAIS
}

\author{
Patrick Leonardo Nogueira da SILVA ${ }^{1}$ \\ Cecília Isabela de Brito Ferreira TEIXEIRA ${ }^{2}$ \\ Elaine Dias SOARES ${ }^{3}$ \\ Carolina dos Reis ALVES ${ }^{4}$
}

\begin{abstract}
${ }^{1}$ Enfermeiro, Especialista em Saúde da Família e Didática e Metodologia do Ensino Superior, Pós-Graduando em Enfermagem do Trabalho, Faculdade de Guanambi/FG. Guanambi (BA), Brasil. E-mail: patrick_mocesp70@hotmail.com

${ }^{2,3}$ Enfermeira, Faculdade Santo Agostinho/FASA. Montes Claros (MG), Brasil. E-mail: cecijanu@ hotmail.com; elaineddias@yahoo.com.br

${ }^{4}$ Enfermeira, Especialista em Saúde da Família, Mestre e Doutoranda em Ciências da Saúde, Professora do Departamento de Enfermagem da Faculdade Santo Agostinho/FASA e Universidade Estadual de Montes Claros/UNIMONTES. Montes Claros (MG), Brasil. E-mail: carolina.calreis@ yahoo.com.br
\end{abstract}

Recebido em: 28/09/2015 - Aprovado em: 17/07/2016 - Disponibilizado em: 18/12/2016

\begin{abstract}
RESUMO
Objetivo: avaliar a qualidade de vida de idosos praticantes de atividade física de uma Unidade Básica de Saúde de Minas Gerais. Métodos: Trata-se de um estudo descritivo, com abordagem quantitativa realizado em uma Unidade de Saúde pública na qual sua amostra compreendeu oito idosos participantes de um grupo de convivência. Utilizou-se como instrumento de coleta de dados o questionário SF-36. O tratamento dos dados se deu por meio de epidemiologia descritiva. O projeto de pesquisa foi aprovado pelo Comitê de Ética em Pesquisa, protocolo $n^{\circ}$ 494.428. Resultados: A maior parte dos idosos era do sexo feminino, idade entre 70-79 de idade, casados, com ensino fundamental e praticam atividade física quatro vezes ou mais por semana. Cada participante teve filhos durante a sua vida e ainda reside com pelo menos um deles, com exceção de uma que reside sozinha. Observou-se que a motivação para a prática de exercícios físicos é diferente entre os dois sexos, sendo que as mulheres são mais ativas. Os idosos relataram se sentirem emocionalmente melhor, mais felizes, quando frequentam o grupo de atividade física. O resultado do estudo apontou que a idade mais avançada pode interferir na qualidade de vida dos idosos na questão da mobilidade e perda da força muscular que além de causarem dor e desconforto atrapalhando a prática de exercício. Conclusão: Portanto, a qualidade de vida de quem pratica atividade física é positiva, juntamente com a percepção do envelhecimento mais saudável, porém a idade avançada pode interferir na qualidade de vida dos idosos quanto à questão da mobilidade e perda da força muscular, pois além de causarem dor nos praticantes de atividade física, podem causar desconforto, transformando em empecilho durante a prática da atividade.
\end{abstract}

Palavras-chave: Idoso. Atividade motora. Qualidade de vida.

\section{EVALUATION OF THE QUALITY OF LIFE OF ELDERLY PRACTITIONERS OF PHYSICAL ACTIVITY OF A HEALTH BASIC UNIT OF MINAS GERAIS}

\begin{abstract}
Objective: To evaluate the quality of life of elderly practitioners of physical activity of a Basic Health Unit of Minas Gerais. Methods: This is a descriptive study with a quantitative approach carried out in a public health unit in which your sample comprised eight elderly participants of a support group. It was used as data collection instrument of the SF-36 questionnaire. Data analysis was done through descriptive epidemiology. The research
\end{abstract}


project was approved by the Research Ethics Committee, protocol n. 494428. Results: Most elderly were female, aged between 70-79 of age, married, with primary and practice physical activity four or more times a week. Each participant had children during his lifetime and still lives with at least one of them, except for one who lives alone. It was observed that the motivation for physical exercise is different between the sexes, while women are more active. The elderly reported feeling emotionally better, happier, when attending the physical activity group. The result of the study found that older age can affect the quality of life of older people on mobility and loss of muscle strength which in addition to causing pain and discomfort hindering the exercise. Conclusion: Therefore, the quality of life of those who practice physical activity is positive, along with the perception of healthier aging, but old age can interfere with quality of life of older people on the issue of mobility and loss of muscle strength, as well to cause pain physically active, they can cause discomfort, turning into obstacle during practice activity.

Keywords: Aged. Motor activity. Quality of life.

\section{INTRODUÇÃO}

O envelhecimento populacional é um fenômeno natural, irreversível e mundial sendo descrito como um conjunto de modificações morfofisiológicas, bioquímicas e psicológicas, que determinam a perda progressiva da capacidade de adaptação do indivíduo ao meio ambiente, sendo considerado um processo dinâmico e progressivo (FERREIRA et al., 2010). A população idosa brasileira vem crescendo de forma acelerada, com previsão de que no ano de 2025, o Brasil seja o sexto país com a maior população de idosos do mundo, com um percentual de aumento de aproximadamente $14 \%$, o que significa, em números absolutos, cerca de 32 milhões de idosos. Esta transição demográfica tem como fator as mudanças nos hábitos saudáveis da população em geral (BRASIL, 2013).

O idoso deve realizar atividades que mantenham em funcionamento o seu sistema neuromotor de modo que possa levar em conta a ajuda de outros para a realização das atividades de vida diária (AVD), tais como mobilidade, alimentação, vestuário, dentre outras, mantendo-se uma pessoa independente e autônoma e, deste modo, levando uma melhor qualidade de vida (QV). Estudos em gerontologia têm demonstrado que a atividade física aliada a outros aspectos tais como hereditariedade, alimentação adequada e hábitos de vida apropriados, podem melhorar em muito a QV dos idosos. Embora existam questionamentos em relação ao papel da atividade física no processo de envelhecimento, há muitos dados que configuram seu benefício incontestável para aqueles que a praticam, em relação à saúde física, mental, psicológica e social (OKUMA, 1998; RODRIGUES et al., 2007; BRASIL, 2010; GUIMARÃES et al., 2012).

$\mathrm{O}$ interesse pelo conceito da QV é relativamente recente e decorre, em parte, 
dos novos padrões que têm influenciado as políticas e as práticas do setor nas últimas décadas. As condições de vida e saúde vêm melhorando de forma contínua e sustentada na maioria países, tudo graças aos progressos dos políticos, econômicos, sociais e ambientais, assim como na saúde pública e na medicina (LIMA-COSTA; VERAS, 2003). A QV é um tema complexo e muito relevante, pois está diretamente relacionado à promoção da saúde e também está relacionada com os seguintes componentes: capacidade física, estado emocional, interação social, atividade intelectual, situação econômica e autoproteção de saúde (SANTOS et al., 2002). Segundo Minayo (2000), QV é uma noção eminentemente humana, que tem sido aproximada ao grau de satisfação encontrado na vida familiar, amorosa, social e ambiental e à própria estética existencial.

$\mathrm{O}$ ato de exercitar-se precisa estar incorporado não somente ao cotidiano das pessoas, mas também à cultura popular, aos tratamentos médicos, ao planejamento da família e à educação infantil. Pois essa necessidade se dá por diferentes fatores: do fator social, quando se proporciona ao homem o direito de estar ativo fisicamente em grupo, ao fator econômico, quando se constata que os custos com saúde individual e coletiva caem em populações fisicamente ativas (PICCOLO, 2011). Segundo Oliveira et al. (2010), a prática de exercício físico, além de combater o sedentarismo, vai contribui de uma maneira significativa para a manutenção da aptidão física do idoso, tanto na sua vertente da saúde, como nas capacidades funcionais. Outra vantagem promovida pela prática de exercícios é a melhora das funções orgânicas e cognitivas, garantindo maior independência pessoal e prevenindo doenças.

Os benefícios promovidos pela prática regular de atividade física são sugeridos como uma alternativa de melhora da QV em idosos. Em alguns estudos transversais, demonstraram que há uma correlação moderada e positiva entre a atividade física e os benefícios para a QV. Uma vez que é importante, não apenas aumentar os anos vividos pelos indivíduos, mas também aumentar concomitantemente a QV, durante esses anos é importante que sejam realizados estudos que avaliem o impacto da prática de atividade física neste desfecho (OLIVEIRA et al., 2010; GUIMARÃES et al., 2012).

$$
\text { Portanto, objetiva-se avaliar a QV }
$$
de idosos praticantes de atividade física de uma Unidade Básica de Saúde (UBS) de Minas Gerais. 


\section{MÉTODO}

Trata-se de um estudo descritivo, exploratório, com abordagem quantitativa. $\mathrm{O}$ estudo teve como cenário o bairro Antônio Pimenta, localizado na cidade de Montes Claros, Minas Gerais (MG). A amostra foi composta por oito idosos que freqüentam o grupo de terceira idade de atividade física do bairro estudado. Os encontros são semanais, coordenados por dois profissionais vinculados à prefeitura do município em questão na qual as atividades ocorrem em local próximo à residência dos idosos, geralmente em uma quadra próximo ao Centro de Saúde (CS) do bairro. Participam do grupo em média 18 pessoas de ambos os sexos. Foram adotados os seguintes critérios de inclusão para participação no estudo: indivíduos com 60 anos ou mais de idade; estar participando assiduamente do grupo por pelo menos três vezes por semana; pertencer ao grupo no mínimo há seis meses; ser cadastrado no CS; e ser encontrado em até três tentativas. Todos os participantes assinaram um Termo de Consentimento Livre e Esclarecido (TCLE) para que a pesquisa fosse realizada.

$\mathrm{O}$ instrumento utilizado para avaliar a QV dos idosos foi o SF-36, sendo este um questionário genérico de Qualidade de
Vida Relacionada à Saúde (QVRS), composto por 11 questões que abordam oito domínios de QV (capacidade física, aspectos físicos, dor, estado geral de saúde, vitalidade, aspectos sociais, aspectos emocionais e saúde mental), sumarizados em dois componentes: físico e mental (WARE; SHERBOURNE， 1992). O questionário foi previamente traduzido, adaptado culturalmente e validado para a população brasileira (CICONELLI et al., 1999). Os escores das escalas são transformados em valores de zero (pior QV) a 100 (melhor QV) (MCHORNEY et al., 1994; WARE; SHERBOURNE, 1992). A finalidade das questões foi transformar medidas subjetivas em dados objetivos, que permitem ser analisados de forma específica, global e responsável (CICONELLI et al., 1999). Os dados coletados foram tabulados e expressos por meio de tabelas, analisados e comparados um a um com a finalidade de avaliar a QV dos participantes. Foi utilizado o Software Excel® 2010, versão 13.0, como programa estatístico para a tabulação dos dados. O tratamento dos dados se deu por meio de epidemiologia descritiva.

Os preceitos éticos foram obedecidos de acordo a Resolução $n^{\circ}$ 466/2012 do Conselho Nacional de Saúde (CNS) na qual regulamenta a pesquisa envolvendo seres humanos. O projeto de 
pesquisa foi apreciado e aprovado pelo

Comitê de Ética em Pesquisa das

Faculdades Unidas do Norte de Minas

(CEP FUNORTE), sob parecer consubstanciado $\mathrm{n}^{\circ} 494.428$.

\section{RESULTADOS}

A maior parte dos idosos era do sexo feminino (75\%), com faixa etária entre 70-79 anos (62,5\%, casado(a) (50\%), com ensino fundamental (50\%), e praticam atividade física por quatro vezes ou mais durante a semana $(87,5 \%)$ (Tabela 1$)$.

Tabela 1 - Perfil socioeconômico dos idosos entrevistados. Montes Claros (MG), Brasil,

\begin{tabular}{|c|c|c|}
\hline Variáveis & $\mathrm{n}=8$ & $\%$ \\
\hline \multicolumn{3}{|l|}{ Sexo } \\
\hline Masculino & 02 & 25,0 \\
\hline Feminino & 06 & 75,0 \\
\hline \multicolumn{3}{|l|}{ Faixa etária } \\
\hline 60-69 anos & 03 & 37,5 \\
\hline 70-79 anos & 05 & 62,5 \\
\hline$>80$ anos & 00 & 00,0 \\
\hline \multicolumn{3}{|l|}{ Estado civil } \\
\hline Solteiro(a) & 00 & 00,0 \\
\hline Casado(a) & 04 & 50,0 \\
\hline Divorciado(a) & 01 & 12,5 \\
\hline Viúvo(a) & 03 & 37,5 \\
\hline \multicolumn{3}{|l|}{ Escolaridade } \\
\hline Analfabeto & 03 & 37,5 \\
\hline Ensino fundamental & 04 & 50,0 \\
\hline Ensino médio & 00 & 00,0 \\
\hline Ensino superior & 01 & 12,5 \\
\hline \multicolumn{3}{|l|}{ Atividade física } \\
\hline Uma vez por semana & 00 & 00,0 \\
\hline Duas vezes por semana & 00 & 00,0 \\
\hline Três vezes por semana & 01 & 12,5 \\
\hline Quatro vezes ou mais & 07 & 87,5 \\
\hline
\end{tabular}

(MG), Brasil, 2014.

Todos entrevistados possuem filhos e residem no mínimo com um dos seus filhos, exceto uma que tem filho, porém, mora só. Dos idosos em questão, todos são participantes de grupos operativos em que um idoso $(12,5 \%)$ participa três vezes na semana e sete $(87,5 \%)$ participam quatro vezes ou mais durante a semana.
De acordo o Quadro 1, o mesmo mostra os dados relacionados com a cognição, sendo esta com e sem declínio, e se há ou não a prática de atividade física, obtidos do preenchimento do questionário de QV. Os dados apontam que, a maioria não apresenta declínio, mas que apresenta 
Quadro 1 - Valores dos domínios avaliados, segundo o questionário FS-36, dos idosos com e sem declínio cognitivo e a atividade física. Montes Claros (MG), Brasil, 2014.

\begin{tabular}{|c|c|c|c|c|c|}
\hline \multirow[t]{2}{*}{ DOMÍNIO } & \multirow{2}{*}{$\begin{array}{l}\text { ATIVIDADE } \\
\text { FÍSICA }\end{array}$} & \multicolumn{2}{|c|}{$\begin{array}{c}\text { COM DECLÍNIO } \\
\text { COGNITIVO }\end{array}$} & \multicolumn{2}{|c|}{$\begin{array}{l}\text { SEM DECLÍNIO } \\
\text { COGNITIVO }\end{array}$} \\
\hline & & $n=8$ & $\%$ & $n=8$ & $\%$ \\
\hline Capacidade funcional & SIM & 03 & 37,5 & 05 & 62,5 \\
\hline 2. $\quad$ Aspecto físico & SIM & 02 & 25,0 & 06 & 75,0 \\
\hline 3. Dor & SIM & 06 & 75,0 & 02 & 25,0 \\
\hline 4. Estado geral de saúde & SIM & 00 & 00,0 & 08 & 100,0 \\
\hline 5. $\quad$ Vitabilidade & SIM & 01 & 12,5 & 07 & 87,5 \\
\hline 6. $\quad$ Aspecto social & SIM & 01 & 12,5 & 07 & 87,5 \\
\hline 7. Aspecto emocional & SIM & 03 & 37,5 & 05 & 62,5 \\
\hline 8. $\quad$ Saúde mental & SIM & 01 & 12,5 & 07 & 87,5 \\
\hline
\end{tabular}

Fonte: Elaboração própria. Montes Claros (MG), Brasil, 2014.

O Quadro 1 ilustra que os idosos sem declínio cognitivo tiveram melhores resultados em todos os domínios na auto percepção da QV com exceção apenas do domínio dor em que os idosos com declínio tiveram maior percentagem haja vista que dor tem relação indireta com QV.

\section{DISCUSSÃO}

$\mathrm{O}$ resultado mostrou que a motivação para a prática de atividade física feminina é diferente da masculina. A maioria das entrevistadas relatou que não tiveram atividade profissional, sempre exerceram o papel de dona de casa, mãe e esposa. A participação das mulheres em grupos de atividade física pode estar relacionada com a feminização da população idosa aliada a disponibilização de tempo, além do fato de que o cuidar é um ato cuja gênese é feminina. Durante o estudo, foi possível perceber que a maioria avaliou a própria $\mathrm{QV}$ como positiva e que a atividade física contribuiu muito para que isso acontecesse de forma a melhorar tanto a parte física quanto a emocional, pois além de trabalhar o corpo, é possível também estabelecer um vínculo emocional muito forte com as amizades feitas durante as atividades.

Os idosos relataram se sentir emocionalmente melhor, mais felizes quando frequentam o grupo de atividade física, pois é no grupo que eles encontram pessoas na mesma situação, trazendo um conforto para o seu emocional, pois, segundo Pereira et al. (2006), a QV reflete a percepção que tem os indivíduos de que suas necessidades estão sendo satisfeitas ou ainda que lhes estejam sendo negadas a oportunidades de encontrar a felicidade. Segundo Araújo (2005), as mulheres vivem em média oito anos a mais que os 
homens. Isso pode ser explicado devido à participação feminina em grupos de atividades na qual esta participação pode está relacionada com as preocupações e manter o bem-estar de um modo geral, quando comparado ao homem idoso. De acordo com Pereira et al. (2006), a atividade física, o estresse a percepção e a aceitação em relação ao envelhecimento estavam fortemente associados à $\mathrm{QV}$ nas mulheres. $\mathrm{O}$ estudo realizado por Leopardi (2002) encontrou resultado idêntico com predomínio de mulheres nas atividades físicas.

No presente estudo o verificou-se que, a predominância de idosos foi de 7079 anos. Para Filgueiras Júnior (2013), quanto maior a idade, pior a $\mathrm{QV}$ dos idosos. Isso ocorre devido ao processo de envelhecimento que traz consigo alterações em todos os órgãos e sistemas do corpo humano. Ainda para o autor, a atividade física, nutrição e outros fatores de estilo de vida têm grande influência sobre a saúde do idoso. Os idosos estudados são, em sua maioria, de baixa escolaridade segundo o Instituto Brasileiro de Geografia e Estatística (IBGE), ou seja, quem tem menos de quatro anos de estudo podem ser considerados analfabetos funcionais (IBGE, 2005). Segundo Pereira et al. (2006), fatores como baixo nível escolaridade estão relacionados a baixo nível de $\mathrm{QV}$, porém, no presente estudo a baixa escolaridade não interfere na QV.

Segundo Guimarães et al. (2004), o envelhecimento é um processo dinâmico e progressivo na qual há alterações morfofuncionais e bioquímicas, com redução na capacidade de adaptação homeostática às situações de sobrecarga funcional, alterando progressivamente $\mathrm{o}$ organismo e tornando-o mais susceptível às agressões intrínsecas e extrínsecas. Nos domínios à atividade física, a maioria dos estudados realizam suas atividades físicas dentro de suas limitações, este estudo evidencia um resultado semelhante ao estudo de Leite et al. (2012), onde avalia as limitações para a realização de AVD e seu impacto na vida do idoso. Pois, na prática de atividade física, o corpo libera hormônios que induzem a excitação, tanto física, quanto mental e de bom humor, que, conseqüentemente, trará benefícios físicos. De acordo com Montovani et al. (2006), a prática regular de exercícios físicos é reconhecida como uma das melhores formas de se prevenir e de combater os males associados com o envelhecimento. A prática de atividade física assegura maior independência, autonomia e melhor condição de saúde, aumentando o senso de bem-estar, a crença de auto-eficácia e a capacidade do indivíduo de atuar sobre o meio ambiente e sobre a si mesmo. 
No domínio relacionado à dor, constatou-se declínio significativo na QV. Nos idosos, a dor é um fator que limita suas funções e prejudica suas atividades diárias. Segundo Leite (2012), a dor pode chegar a casos mais acentuados, tal como o isolamento social, até por que a dor é uma experiência sensorial e emocional tida como ruim. No presente estudo identificou-se também que, idosos com idade mais avançada têm certa dificuldade para exercer suas AVD sendo a dor o empecilho, resultado similar ao estudo feito por Andrade (2006), para um diagnóstico preciso de dor no idoso, especialmente entre aqueles com idade mais avançada, recomenda-se que a avaliação seja feita de forma exaustiva, exame físico minucioso e avaliação do estado funcional, psíquico e social, cuidando para não se restringir apenas ao auto-relato do paciente. Ainda de acordo Andrade (2006), a dor pode estar associada a imagens negativas, tais como sofrimento prolongado, transtornos psiquiátricos, inexistência de tratamento e abuso de medicamentos, tornando-se um problema para o indivíduo, a família e a sociedade, uma vez que direciona e limita as condições e o comportamento daquele que a vivencia, aumentando a morbidade.

Nos domínios do estado geral de saúde e vitalidade, o resultado mostrou que, em sua maioria os idosos se sentem bem, cheios de força para realizar suas atividades físicas. $\mathrm{O}$ que acaba tendo uma lógica, na medida em que, maior é a vitalidade com a realização de atividade física, conseqüentemente melhor será o seu estado geral de saúde. Os resultados mostraram que a saúde dos entrevistados teve uma melhora significativa com a prática de atividade física, resultado semelhante ao estudo feito por Maciel (2010), onde mostra que relação aos cuidados prévios à prática das atividades físicas, recomenda-se a realização das mesmas somente quando houver bem-estar físico por parte do idoso. No quesito social e emocional, os estudados relataram que não deixam o estado emocional interferir na sua vida social, pelo contrario, eles vão ao grupo de atividade física para manter o bem-estar físico e manter também o bem estar psicológico. Ainda sobre este domínio, foi uma das melhores médias, onde o grupo de convivência freqüentado pelos estudados é a fonte que os proporcionam felicidade, vontade de viver e conforto diante das suas limitações.

Este estudo é evidenciado pelo estudo feito por Wichmann (2013), onde a satisfação com a vida é uma das medidas do bem-estar psicológico, que reflete a avaliação pessoal do indivíduo sobre determinados domínios. As redes de 
relações são importantes fontes de suporte social e estão relacionadas ao senso de bem-estar. Do mesmo modo, o nível de satisfação dos idosos na convivência com outras pessoas pode aumentar de intensidade no decorrer da vida, melhorando a QV. Nos grupos, surge a oportunidade de estabelecer novas amizades, ampliar os conhecimentos e afastar a solidão.

Segundo Ferreira et al. (2010), a Organização Mundial de Saude (OMS) destacou que a participação dos idosos em atividades físicas leves e moderadas, como um importante fator no retardo dos declínios funcionais, melhorando a sua saúde motora e mental. O mesmo pode ser evidenciado na presente pesquisa que mostrou a independência dos idosos em resolver seus próprios problemas sem a interferência de familiares e/ou cuidadores. Durante a pesquisa foi possível perceber que a maioria dos idosos avaliou a própria qualidade de vida como positiva e que a atividade física contribuiu muito para esta melhora. Conforme Oliveira et al. (2010), o mesmo destaca que a positividade também é percebida na questão física e emocional, pois além de trabalhar a parte do corpo é possível também estabelecer um vínculo emocional muito forte entre os participantes.

\section{CONCLUSÃO}

Observou-se o número maior de mulheres de forma a caracterizar uma maior preocupação com a saúde neste gênero. A população integrante do grupo de atividade física é assídua, o que indica que há um investimento dos membros do grupo com a atividade. Infere-se ainda que a QV dos praticantes de atividade física é positiva, juntamente com a percepção do envelhecimento e a vontade de viver. Sabese que, durante $\mathrm{o}$ processo de envelhecimento e durante a vida, ocorrem perdas tanto física como na capacidade funcional, sendo oportuno o investimento em programas que retardem essas perdas, para que o envelhecimento possa ser aproveitado e vivido da melhor forma possível. O estudo mostrou que a idade avançada pode interferir na $\mathrm{QV}$ dos idosos quanto à questão da mobilidade e perda da força muscular, pois além de causarem dor nos praticantes de atividade física, podem causar desconforto, transformando em empecilho durante a prática da atividade.

\section{REFERÊNCIAS}

ANDRADE, F. A.; PEREIRA, L. V.; SOUSA, F. A. E. F. Mensuração da dor no idoso: uma revisão. Revista LatinoAmericana de Enfermagem. Ribeirão Preto, v. 14, n. 2, p. 271-276, 2006. Disponível em: 
http://www.scielo.br/pdf/rlae/v14n2/v14n2 a18.pdf. Acesso em: 21 mar 2014.

ARAÚJO, L. F.; COUTINHO, M. P. L.; CARVALHO, V. A. M. L. Representações sociais da velhice entre idosos que participam de grupos de convivência.

Psicologia: Ciência e Profissão. Brasília, v. 25, n. 1, p. 118-131, 2005. Disponível em:

http://www.scielo.br/pdf/pcp/v25n1/v25n1 a10.pdf. Acesso em: 21 mar 2014.

BRASIL. MINISTÉRIO DA SAÚDE. SECRETARIA DE ATENÇÃO À SAÚDE. DEPARTAMENTO DE AÇÕES PROGRAMÁTICAS E ESTRATÉGICAS.

Atenção à saúde da pessoa idosa $\mathrm{e}$ envelhecimento. Brasília (DF), 2010.

Disponível em:

http://bvsms.saude.gov.br/bvs/publicacoes/ atencao_saude_pessoa_idosa_envelhecime nto_v12.pdf. Acesso em: 28 mai 2014.

BRASIL. MINISTÉRIO DA SAÚDE. INSTITUTO DE ESTUDOS DE SAÚDE SUPLEMENTAR. Envelhecimento populacional e os desafios para o sistema de saúde brasileiro. São Paulo: IESS,

2013. Disponível em:

http://www.iess.org.br/html/1apresentao.pd f. Acesso em: 22 set 2015.

CICONELLI, R. M.; FERRAZ, M. B.; SANTOS, W.; MEINÃO, I.;

QUARESMA, M. R. Tradução para língua portuguesa e validação do questionário genérico de avaliação da qualidade de vida SF-36 (Brasil SF-36). Revista Brasileira de Reumatologia. São Paulo, v. 39, n. 3, p. 143-50, 1999. Disponível em: http://www.ufjf.br/renato_nunes/files/2014 /03/Valida\%C3\%A7\%C3\%A3o-doQuestion\%C3\%A1rio-de-qualidade-deVida-SF-36.pdf. Acesso em: 28 mai 2014.

FERREIRA, O. G. L.; MACIEL, S. C.; SILVA, A. O.; SANTOS, W. S.;

MOREIRA, M. A. S. P. Active aging from the perspective of aged individuals who are functionally independent. Revista da

Escola de Enfermagem da USP. São Paulo, v. 44, n. 4, p. 1060-1064, 2010. Disponível em:

http://www.scielo.br/pdf/reeusp/v44n4/en 30.pdf. Acesso em: 24 mar 2014.

FILGUEIRAS JÚNIOR, N. Qualidade de vida dos atletas da terceira idade do município de Ponta Porã, MS. 2013. 93 f. Dissertação (Mestrado em Psicologia) Programa de Pós-Graduação em Psicologia, Universidade Católica Dom Bosco, Campo Grande. Disponível em: http://site.ucdb.br/public/mddissertacoes/13200-final.pdf. Acesso em: 28 mai 2014.

GUIMARÃES, L. H. C. T.; GALDINO, D. C. A.; MARTINS, F. L. M.; VITORINO, D. F. M.; PEREIRA, K. L.; CARVALHO, E. M. Comparação da propensão de quedas entre idosos que praticam atividade física e idosos sedentários. Revista

Neurociências. São Paulo, v. 12, n. 2, p. 68-72, 2004. Disponível em:

http://www.revistaneurociencias.com.br/ed icoes/2004/RN\%2012\%2002/Pages\%20fro m\%20RN\%2012\%2002-2.pdf. Acesso em: 30 mar 2014.

GUIMARÃES, A. C. A.; SCATTI, A. V.; SOARES, A.; FERNANDES, S.;

MACHADO, Z. Percepção da qualidade de vida e da finitude de adultos da meia idade e idosos praticantes e não praticantes de atividade física. Revista Brasileira de Geriatria e Gerontologia. Rio de Janeiro, v. 15, n. 4, p. 661-670, 2012. Disponível em:

http://www.scielo.br/pdf/rbgg/v15n4/07.pd f. Acesso em: 25 mar 2014.

INSTITUTO BRASILEIRO DE GEOGRAFIA E ESTATÍSTICA. Síntese de indicadores sociais, 2004. Rio de Janeiro: IBGE, 2005. Acesso em: 28 mai 2014.

LEITE, M. T.; WINCK, M. T.; HILDEBRANDT, L. M.; KIRCHNER, R. 
M.; SILVA, L. A. A. Qualidade de vida e nível cognitivo de pessoas idosas participantes de grupos de convivência.

Revista Brasileira de Geriatria e Gerontologia. Rio de Janeiro, v. 15, n. 3, p. 481-492. 2012. Disponível em: http://www.scielo.br/pdf/rbgg/v15n3/v15n 3a09.pdf. Acesso em: 20 mar 2014.

LEOPARDI, M. T. Alguns aspectos da pesquisa quantitativa. In: LEOPARDI, M. T. (Orgs). Metodologia da pesquisa na saúde. Florianópolis: UFSC, 2002. Acesso em: 1 nov 2014.

LIMA-COSTA, M. F.; VERAS, R. Saúde publica e envelhecimento. Cadernos de Saúde Pública. Rio de Janeiro, v. 19, n. 3, p. 700-701, 2003. Disponível em: http://www.scielo.br/pdf/csp/v19n3/15872. pdf. Acesso em: 21 mar 2014.

MACIEL, M. G. Atividade física e funcionalidade do idoso. Motriz. Revista de Educação Física. Rio Claro, v. 16, n. 4, p. 1024-1032, 2010. Disponível em: http://www.periodicos.rc.biblioteca.unesp. br/index.php/motriz/article/view/19806574.2010v16n4p1024/pdf_23. Acesso em: 22 mar 2014.

MANTOVANI, R. Idosos em movimento: reflexões e históricas sobre a mobilidade na terceira idade. São Paulo, 2006. Acesso em: 28 mai 2014.

McHORNEY, C. A.; WARE JUNIOR, J. E.; SHERBOURNE, S. D. The Mos 36Item Short-Form Health Survey (Sf-36): III. Tests of data quality, scaling assumptions and reliability across diverse patient groups. Medical Care.

Massachusetts, v. 32, n. 1, p. 40-66, 1994. Disponível em:

http://www.ncbi.nlm.nih.gov/pubmed/8277 801. Acesso em: 28 mai 2014.

MINAYO, M. C. S.; HARTZ, Z. M. A.; BUSS, P. M. Qualidade de vida e saúde: um debate necessário. Ciência \& Saúde
Coletiva. Rio de Janeiro, v. 5, n. 1, p. 7-18, 2000. Disponível em:

http://www.scielo.br/pdf/csc/v5n1/7075.pd f. Acesso em: 22 mar 2014.

OKUMA, S. S. Idoso e a atividade física. Campinas (SP): Papirus, 1998. Acesso em: 28 mai 2014.

OLIVEIRA, A. C.; OLIVEIRA, N. M. D.; ARANTES, P. M. M.; ALENCAR, M. A. Qualidade de vida em idosos que praticam atividade física - uma revisão sistemática.

Revista Brasileira de Geriatria e Gerontologia. Rio de Janeiro, v. 13, n. 2, p. 301-312, 2010. Disponível em: http://www.scielo.br/pdf/rbgg/v13n2/a14v 13n2.pdf. Acesso em: 24 mar 2014.

PEREIRA, R. J.; COTTA, R. M. M.; FRANCESCHINI, S. C. C.; RIBEIRO, R. C. L.; SAMPAIO, R. F.; PRIORE, S. E.; CECON, P. R. Contribuição dos domínios físico, social, psicológico e ambiental para a qualidade de vida global de idosos.

Revista Psiquiatria do Rio Grande do

Sul. Porto Alegre, v. 28, n. 1, p. 27-38, 2006. Disponível em:

http://www.scielo.br/pdf/rprs/v28n1/v28n1 a05.pdf. Acesso em: 28 mai 2014.

PICCOLO, G. M. Os caminhos dialéticos do envelhecimento e sua relação com a educação física contemporânea. Revista Brasileira de Geriatria e Gerontologia. Rio de Janeiro, v. 14, n. 1, p. 169-177, 2011. Disponível em: http://www.scielo.br/pdf/rbgg/v14n1/a17v 14n1.pdf. Acesso em: 22 set 2015.

RODRIGUES, R. A. P.; KUSUMOTA, L.; MARQUES, S.; FABRÍCIO, S. C. C.; CRUZ, I. R.; LANGE, C. Política nacional de atenção ao idoso e a contribuição da enfermagem. Texto \& Contexto -

Enfermagem. Florianópolis, v. 16, n. 3, p. 536-545, 2007. Disponível em: http://www.scielo.br/pdf/a21v16n3.pdf. Acesso em: 28 mai 2014. 
SANTOS, S. R.; SANTOS, I. B. C.;

FERNANDES, M. G. M.; HENRIQUES,

M. E. R. M. Qualidade de vida do idoso na

comunidade: aplicação da escala de

Flanagan. Revista Latino-Americana de

Enfermagem. Ribeirão Preto, v. 10, n. 6, p. 757-764, 2002. Disponível em:

http://www.scielo.br/pdf/rlae/v10n6/v10n6

a2.pdf. Acesso em: 21 mar 2014.

WARE JUNIOR, J. E.; SHERBOURNE,

$S$. D. The MOS 36-item short-form health survey (SF-36). I. Conceptual framework and item selection. Medical Care.

Massachusetts, v. 30, n. 6, p. 473-483, 1992. Disponível em:

http://www.ncbi.nlm.nih.gov/pubmed/1593 914. Acesso em: 28 mai 2014.

WICHMANN, F. M. A.; COUTO, A. N.; AREOSA, S. V. C.; MONTAÑES, M. C. M. Grupos de convivência como suporte ao idoso na melhoria da saúde. Revista

Brasileira de Geriatria e

Gerontologia. Rio de Janeiro, v. 16, n. 4, p. 821-832, 2013. Disponível em: http://www.scielo.br/pdf/rbgg/v16n4/18099823-rbgg-16-04-00821.pdf. Acesso em: 21 mar 2014. 